Aside from the work of Weber and Wahlfors, I think I am the only man who has done any experimental work on living human eyes in situ, by connecting the interior of the eye to a manometer.

\title{
THE VISUAL PERCEPTION OF SOLID FORM.*
}

\author{
BY \\ E. M. Eaton. \\ (Concluded from p. 340)
}

In the circumstances which lead to the perception of double images a small amount of non-identity of direction can be overcome by the tendency to achieve single vision. This is accomplished by error of projection, and the limits within which it is possible are those which have been described as the limits of fusion. As the underlying cause is in effect illusion of form, they are probably closely associated with the limits of illusion.

The mechanism of binocular vision, as I conceive it, may be summarized as follows:

1. The vision of each eye remains physiologically independent except as regards fixation; apart from this the unification of the two images has reference to the object perceived not to the means of perception.

2. The physiological axes of vision are interpreted as occupying their actual positions, the lines passing through the point of fixation and the nodal points of each eye.

3. The perceptive axis of vision lies between the fixation point and the middle of the interocular space. Its use involves a psychic modification of all visual angles similar to that which enables us to interpret foreshortened images in accordance with their true values.

4. The perception of position in three dimensions is a function of the sense of directional projection of the two eyes. The perception of relative position in three dimensions is a function of the sense of relative directional projection of the two eyes.

5. Double images result from a certain degree of perception of the subjective element in the sense of sight, with consequent alteration of the foregoing relationships.

If binocular impressions are but a phase, of projection sense impressions, or more inclusively of form sense impressions, there can be no subjective reason, no reason on the ground of a specific feeling quality, why the vision to which they give rise should differ

\footnotetext{
* A paper read before the Ophthalmological Section, Royal Society of Medicine,
} on June 4, 1919. 
in category from that afforded by other actions of the form sense; no reason, that is, why the former exclusively should excite stereoscopic consciousness. If there is no subjective reason may there be any objective reason? It is said that by means of binocular vision we see around things, we see the spaces behind them.

We, of course, cannot in the ordinary sense of the word see spaces, yet we may in effect do so to some extent. Those who have looked across a desert plain beneath a tropical mid-day sun will have noticed the appearance of ceaseless motion in the air due to the ever altering states of its refraction. In such circumstances the atmosphere has a very definite effect on our consciousness of spaces. If we look at the objects on the bottom of a pond through a few feet of water the conditions of lighting and reflection are characteristic, and if we are conscious of the medium we must thereby be assisted in our consciousness of the space which it occupies. The variations in the effect of the atmosphere at short distance do not in ordinary circumstances appear to influence our perceptions, but anything which can sufficiently intensify or appear to intensify this factor may affect our vision.

To say that we can see the spaces behind objects is a different matter however. It depends on the question whether it may be possible to see behind an object at all. If the obstructing object is broader than the interocular distance we shall not see more of the object in the background than we would with a single eye if we were at a greater distance; in other words the factor can then be at the most one of degree only, and not of type. If an obstructing -object is narrower than the interocular space, and especially if it is so narrow that our lines of central vision can meet behind it, then provided the conditions cause us to notice that we do see behind it, the effect is that we see through it; we also get double images, one of which may be suppressed. Each image of the intervening body will be seen with one eye, and the background behind it with the other. As a result we shall get transparency of the obstructing abject, for the lines of psychic projection of the background pass through its images. This can be observed with a pencil against a printed page. Under no circumstances do we see around an object, nor do we see behind it in any but the special case of false projection with transparency, and this is obviously not stereoscopic vision.

I have already referred to the influence of looking at objects through water, and the characteristic lighting which results from it. A similar effect is commonly seen in stereoscopic pictures. The view looks as if bathed in an atmosphere approaching in its effect to that of submersion in water. This is due to stereoscopic lustre. The appearance is produced by the superposing of a light 
surface on a shaded one, and with the exaggerated displacement* which is used in the making of our stereoscopic pictures this occurs to an undue extent. It adds beauty to the view, a result which is incidental, and it is one of the reasons that the study of those pictures is apt to make us attribute so much importance to binocular vision.

It sharpens the margins of objects and tends to cut them more clearly apart from the objects behind by suggesting the effect of atmosphere in the vacant spaces, while other factors determine the actual position at which each object will be placed. Binocular vision is in this manner an assistance to stereoscopic vision, although not so greatly in observation of solid bodies as of photographs, for in the former case much more of the lustre of objects is appreciable with one eye.

Stereoscopic lustre is commonly attributed to rivalry of the sensations of light and dark on the two retinae, and to consequent oscillations of light perception; from this it follows that it differs physiologically from lustre as appreciable with one eye. It may be explained on a much more definite basis however.

An object exhibits lustre, or shines, when it gives off light apart from that which we interpret as the visible existence of the object itself. The physical cause commonly underlying this differentiation is regular reflexion, as distinguished from diffusion or irregular reflexion. With regard to perception, the light emitted by objects, excluding for the moment those that are self-luminous, may be divided into two categories, according as it appears to us as an inherent character of the object $x_{x i}$ or as a reflex which, owing to our experience of the variabilty of reflex light, carries the suggestion of extraneous origin. In the former case there is no consciousness of light as such; in the latter there is.

The light which is observed to be of reflex origin, although it is felt as something additional to the fixed identity of the object itself, is none the less referred to the object as medium in ordinary circumstances ; it thus plays a part in our perception of the object, and in the case of clean metallic surfaces it is almost the sole means of perception. This produces the peculiar character of metallic lustre; hence the reflection of white light in sufficient quantity from a black surface, or from an otherwise vacant one like that of a totally reflecting prism, will exactly simulate the appearance of polished silver.

The characteristic quality of the lustre of metals depends upon

- The geometrical effect of excessive distance between the cameras is to make a view representing a reduced model of the subject. This effect, however, is associated only with the degree of dissimilarity of the images, a factor sufficiently small that it is readily overcome by the other guides to size and distance such as the presence of objects of approximately known size, just as similarity of images can be overcome and a depth estimate maintained. 
low absorptive power and high degree of opacity of the lustrous surface. This causes a bright reflex and allows of no transmission of diffused light from irregularly disposed particles beneath the surface.

As it is possible to refer the variable or reflex light to an external source, it follows that under certain circumstances metals ought to be invisible, and this is in fact true. A piece of freshly broken metal shines for the unevenness of its surface does not cause general diffusion but rather multiplication of reflexes; if we polish it moderately it shines more, for we can see more of the reflexes simultaneously; but if we polish it highly there comes a limit when, regarded in a certain way, it ceases to shine, it becomes a mirror; and a perfect mirror has no lustre, it is invisible. The images formed by means of a mirror may themselves shine of course. If we direct the attention to the plane of a mirror, however, using its visible borders or any defects on its surface as guides, we can then regard it as we ordinarily do a piece of metal, and it becomes possessed of the very acme of metallic lustre: the difference between this and invisibility is purely one of mental attitude.

In the case of polished metallic objects in general the indications of contour and surface afforded by the reflexes, serve as an ample basis of definite perceptions, and the almost total absence of diffused light completes the requirements for the perception of metal. The light of self-luminous bodies, of flames, electric lamp filaments, and the stars, resembles reflected light owing to its being intensely brilliant relatively to the surroundings : such bodies thus appear like highly illuminated metallic objects.

Lustre is not due to failure to focus light on the retina for we may accurately focus the light from a mirror by looking at the images beyond it and, while so doing, by attending to the mirror itself we can still accept the interpretation of lustre; similarly with an electric lamp we may focus the filaments accurately, but they remain still lustrous. When lustre is uneven it may cause a sparkling effect; this is sometimes confused with lustre itself, but it is merely a frequent accompaniment or variety.

Lustre is not due to special physical properties of the light, for we may have a choice whether we shall see it or not. In the case of the metals, the imperfections and the form of the surfaces ordinarily so modify the distribution of the reflected light that we have not the choice that is available in the special instance of mirrors : in their particular case, however, the same light appears as lustre or as material reality according as we think of it in relation to the mirror or to the images; in relation to the former it is perceived as reflex light, in relation to the latter it is not differentiable from the objective being of the images themselves. 
When we look at a painting of a soap-bubble, suggestion or voluntary decision determines whether we see lustre or white, blue, green, and other colours of paint.

Lustre is not a specific physiological sensation, it is a specific psychological interpretation. It depends upon the conception of light as a separable idea from that of the object with which we associate it, a conclusion resulting from one or another cause of apparent incongruity of light intensities.

When we combine in the stereoscope a black figure with a white counterpart, we get an image varying somewhat in its shade of grey, as the attention sways more to this side or to that, but all the while shining. Now if the intensity of light falling on the two retinae differs in degree, the perception of brightness is less than that which would be perceived through the more illuminated eye were it acting alone, a phenomenon which is known as Fechner's paradox. It follows that in a stereoscopic combination where there is marked difference in brightness of the two images there is a superfluity of light on the retina which receives the brighter image; there is light beyond that which is interpreted as the visible existence of the object itself, therefore the object shines; in the case of a black and white combination the conditions are analogous to those we have seen to be requisite for the perception of a metallic surface.

The underlying cause of the uniocular and the binocular perception of lustre is thus identical.

The only information of a specific type made available by the binocular factor, consists of certain angular relationships determinable by means of the projection sense. But the interpretation of binocular projection sense impressions requires as a preliminary the correlation of the projection systems of the two eyes. In some circumstances, as in the falling beads experiment or in the observation of points of light in the dark, this is achieved by the attributing of identical origin to the images occupying the foveae. If we block out both foveal images, however, as by holding a pencil transversely about an inch in front of the eyes, we still see in the upper and lower parts of the peripheral field with the full efficiency of our binocular vision. The correlation can now take place only by the comparison of form sense impressions which, as the images are not identical, implies the assumption of their objective significance, and this in its turn involves a reference to preconceived form. This is probably the more common means of correlation, for ordinarily our attention is not confined to the actual point of fixation. It will be observed that the essential difference between foveal and eccentric lines of co-ordination is that in regard to the former, the visual angles are subject to no psychical modifications. When the value of the visual angles is zero, 
differences of proportion due to foreshortening of images are without influence. Hence the line of foveal vision is the line of reference; whether available for actual vision or not, it is the axis in relation to which eccentric visual lines must first be coordinated before they can acquire the perceptive significance necessary for their truthful application in correlating the vision of the two eyes.

Now we have in the sense of perspective-that is, mathematical perspective-another means of determining position in the third dimension, through the relationship of form sense impressions to preconceived form.

From early times the sense of perspective has been recognized by painters as contributing to the effect of relief. For many years it has heen accepted as one of the criteria of distance, that is as a factor in the sense of depth, which, although it is but relief considered in the opposite direction, seems to imply a little more. In the early days of photography it was noticed that photographs made with long focus lenses gave this effect more fully than those made with lenses of higher refraction. This is due to our receiving a more correct perspective impression, which can be obtained with exactitude only if we view the photograph from the position of the lens with which it was made.

The principles governing the obtaining of correct perspective from photographs were first enunciated by Gullstrand, and a specially constructed periscopic lens for realizing the conditions has been designed by von Rohr* at his suggestion. The effect obtainable with it is very realistic even with a single eye.

Although we get true perspective from the distance of the focal length of the lens, this does not of itself explain why we get greater stereoscopic effect. When we view a photograph from too great a distance the effect on the perspective from a geometrical point of view is one of two things : either the distances in depth are exaggerated, or else objects further forward are enlarged relatively to objects further back. The former alternative therefore ought to increase the relief, the latter to cause distortion of the subject. We do not get increased relief in looking at a photograph in the ordinary way, however, we lose in relief; neither do we get distortion. If we are critical we are displeased with the perspective certainly, and if we are able to analyse it we know that the proportions are wrong, but we have no illusion as to the true shapes of the objects represented.

We recognize the picture as a photograph, which, in order that it should give a symmetrical impression, must be looked at from a view-point other than that which we occupy. The retinal image

* von Rohr, Brit. Journ. Photog. Almanac, 1905. 
to which it gives rise, therefore, does not comply with the requirement of being, according to our habit, characteristic of a solid object. Consequently we do not see solidity. We see simply a photograph.

As it is the sense of form which is appealed to in both the binocular sense and in the sense of perspective, we might anticipate that they would be equal in stability, but they are not so. The degrees of difference of directional projection with which the sense of perspective has to deal are much greater than those available to binocular vision, except in the case of very small objects. A much greater error of projection is therefore necessary to permit of its being deceived; it is less susceptible to illusion.

This may be illustrated by an example.

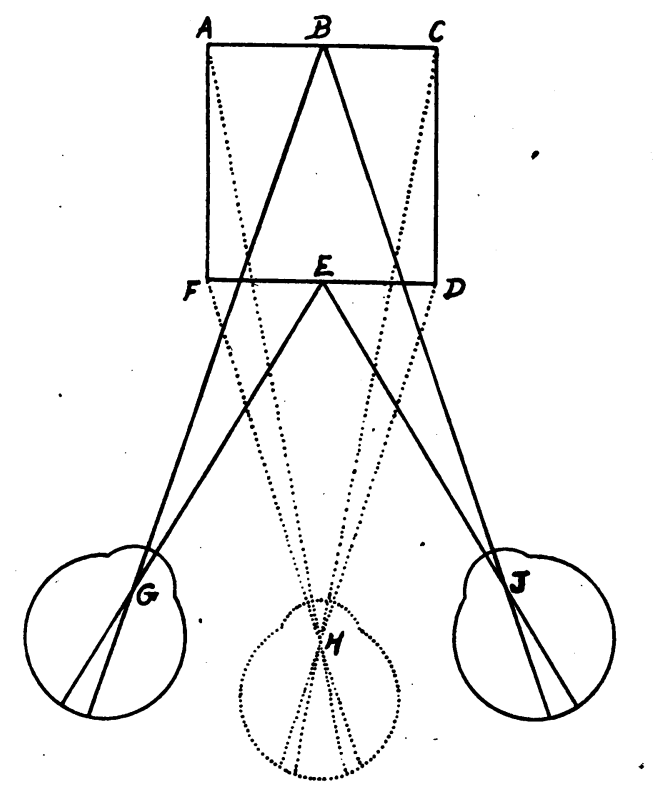

FIG. 6.

A F D C represents a square or skeleton cube of one foot breadth, seen at a distance of 10 feet from its nearer side; and we may represent the binocular view of two points $E$ and $B$ by the continuous lines in the figure, and the uniocular perspective view of the corners by the dotted lines. The angles concerned are $\mathrm{E} \mathrm{G} \mathrm{B}$ and $\mathrm{E} \mathrm{J} \mathrm{B} \mathrm{in} \mathrm{the} \mathrm{former} \mathrm{case,} \mathrm{and} \mathrm{A} \mathrm{H} \mathrm{F} \mathrm{and} \mathrm{D} \mathrm{H} \mathrm{C}$ in the latter. Drawn as in the figure the object is small relatively to the interocular distance, therefore, the perspective angles are the smaller, but if it had been drawn to scale on the measurements given, the angles for perspective would have been 16 minutes, and 
for binocular vision 3 minutes. The larger the object the greater the ratio in favour of perspective; for rectangular shapes the proportions are approximately as the breadth of the object is to the interocular distance. Consequently in average circumstances in the streets of a town, or in the rooms of our houses, the sense of perspective is a much more certain, more useful, and more commanding factor than binocular vision. There is, therefore, every reason on theoretical grounds to expect its part in producing the quality of stereoscopic vision to be greater also. If we take converse circumstances where the object is very small, as in the favourite example of the threading of a needle, then perspective acts so feebly as to be no material assistance, and binocular vision becomes of paramount importance.

There are many other factors which contribute to the building up of our solid visual images; and while I have spoken chiefly of the sense of form or relative projection, I do not wish to give the impression of attaching little importance to factors dependent on other fundamental functions of our sense of sight. I have excluded also all questions of eye movements for, while they are of great value, it is universally accepted that stereoscopic vision is possible without them.

I have here a number of stereoscopic photographs, some of which are mounted normally, others are reversed, and a few are duplicates. They are intended to show the great ease with which the binocular sense will give way in favour of the more imperative demands of the other factors, in particular those of the sense of perspective. If one of the reversed pictures is examined it will be found that although there is some discomfort of vision the appearances are practically normal, but if it is turned top to bottom the objects in the foreground will tend to pass to the back, and vice versa. The reason is that the sense of perspective at first fails to apprehend the meaning of the picture, and is in abeyance, so that the binocular function is free to make the reversal which in the erect position also it would do were it not for the conflict of other and greater evidences.

I have also some ordinary pictorial postcards; these should be viewed with a single eye through $\mathrm{a}+8 \mathrm{D}$ lens to get the natural perspective effect. This principle is not a new one, and the ordinary biconvex lens is in fact a very imperfect one for the purpose, but I show the pictures in order to illustrate the possibility of getting stereoscopy with one eye. I know that there may be differences of opinion whether this is achieved; some will agree that the view is stereoscopic and some may feel that while it is much improved something is wanting as compared with the binocular view.

I would repeat, however, that in order to obtain stereoscopic perception the visual impression should be characteristic of solid 
objects, and that the observation of these characteristics should be habitual. The single correct perspective view fulfils the first condition, but only in part does it fulfil the second condition for people accustomed to use two eyes.

The question is not whether binocular vision is a factor in stereoscopic vision, that is accepted; but is it essential ? The ultimate test must be against solid objects; photographs at the best are but imperfect imitations and the incidental factor of lustre is difficult to eliminate. With reversed pictures, however, the effect of stereoscopic lustre is present as in the normally placed ones; hence those pictures are often more effective than duplicates, despite the greater antagonism of the binocular projection sense. This fact is an index of the degree in which the binocular contribution to stereoscopic appearance is due to this condition, a condition which derives much of its importance from the incidental circumstance that our photographs fail to reproduce natural lustre convincingly.

If we compare the uniocular and binocular perception of a book as seen at a few feet distance on a table; if we choose any view that does not place one or other set of factors in unusual predominance, and compare the two effects; is there any difference in the category of visual feeling which results? In my experience there is not.

The uniocular factors in stereoscopic vision are sometimes spoken of as the psychological factors. Binocular vision is no less a psychological factor, analogous in its mental relationships to the function of perspective vision. Whether we hold the view that perception is unconscious inference, or, that which probably most of us prefer, that it is a mental act of a more immediate type, there would appear to be no ground to differentiate between the perception of flat objècts and the perception of solid ones, between the perception of solids objects by one means and that by another. Each is the product of a sense impression and past experience.

If from a natural view we eliminate experimentally its colouring, its shadow effects, or any other of the stereoscopic guides which it affords, our mental image will lose some little part of its exquisite realism. If, then, we eliminate our binocular vision we shall expect the view to fall in some degree in quality, but we do not lose the sensation of solidity, and that is the criterion. The binocular element in vision contributes a valuable quota to stereoscopic perception, but it is one of many factors. The relative part played by each factor varies in degree with the type of view, and no individual factor is universally essential.

If from the retinal stimulus, and consequently from our physical impression, we take away any one of the features of solidity, then the sensation is less vivid, but the perception of form does not vary. 
The absence of binocular vision is a disadvantage, but I doubt if this is so great as that involved in a reduction of the sense of light difterence which cuts down the shadow effects.

The ultimate requirement in the perception of solid form is that the retinal stimuli must present a certain minimal amount of the characteristics of solidity to which the individual is accustomed, such as perspective, shadow effects, graded definition, and, in the case of those with two eyes, the two differing images. Where these stimuli are provided by photographs or other projection representations, it is necessary also that they do not contain inconsistences greater than will, in the particular circumstances, be overcome $b_{j}^{y}$ the accommodative elasticity of wbatever sense factors may be concerned.

\title{
THE MONTGOMERY LECTURE. I9I7-I9I8*
}

\section{A survey of cases of concomitant squint in the practice of the late Mr. P. W. Maxwell.}

\author{
BY \\ Euphan M. MAXWell, M.B:, \\ DUBLIN. \\ (Concluded from page 349.)
}

' V. Operative technique and results.-Mr. Maxwell's operative technique may be thus briefly outlined:

(1) His tenotomies were always "complete," the tendon being severed as far forward as possible; the conjunctival wound was invariably sutured. The introduction of an "emergency suture" was a later development. This consisted of a double-threaded suture passed from behind forwards through the divided tendon and anterior lip of the conjunctival wound. It was left ready to tie over a bead, within a few hours to a few days after the operation, if an over effect was noted.

(2) His shortenings were performed with a "tucker" of his own design (Fig. 1).

Screw "a" regulates the height to which the control hook can raise the muscle. In the figure it stands about $8 \mathrm{~mm}$. above the lateral hooks, in which position a shortening of $16 \mathrm{~mm}$. could be accomplished.

Fig. 2 illustrates the tucker in position.

The double suture seen held in the forceps, was first passed through the posterior lip of the conjunctival wound, then through

*Delivered in the School of Physic, Trinity College, Dublin, on Friday, Nov. 8, 1918. 